\title{
Victimización y perpetración de violencia en pareja adolescente y redes de apoyo en Colombia. Análisis con perspectiva de género
}

\author{
Johanna Alexandra Reina-Barreto \\ Doctora en Trabajo Social. Trabajadora Social \\ Universidad Complutense de Madrid. Madrid, España \\ https:/ / orcid.org/0000-0001-5541-986X • johanna.a.reina@gmail.com
}

\section{Resumen}

Este estudio analiza la victimización y perpetración de violencia en relaciones de pareja adolescente así como otras variables sociodemográficas comparando al grupo que buscó apoyo social por esta violencia con el grupo que no buscó apoyo. Describe, además, la estructura y densidad de dichas redes de apoyo a partir de una muestra conformada por 2.217 mujeres de entre 13-19 años que respondieron la macro-encuesta de salud Bogotá2011.Se utilizaron tablas de contingencia con contraste Chi-cuadrado y el coeficiente V de Cramer para comprobar la intensidad de la relación entre variables.

En victimización, el 9\% de la muestra sufrió violencia por su pareja masculina y de ellas, el 67,5\% no buscó apoyo. El grupo que buscó apoyo informó hasta 19 formas distintas de violencia reportadas por una misma adolescente. A mayor prevalencia e intensidad de la violencia mayor búsqueda de apoyo, sin bien convivir con el agresor disminuyó dicha búsqueda. En perpetración, el 3,2\% de la muestra ejerció violencia hacia su pareja masculina agresora. Las adolescentes que más violencia sufrieron no ejercieron violencia perpetraron hacia sus parejas agresoras en mayor proporción. Respecto a las redes de apoyo, la familia fue la principal fuente. Los resultados indican que las mujeres adolescentes experimentan violencia de género en sus relaciones de pareja y que la dominación masculina continúa presente, invisibilizada y legitimada culturalmente en las nuevas generaciones. Se evidencia la importancia de diseñar desde el Trabajo Social políticas sociales e intervenciones que contribuyan a deconstruir las relaciones desiguales antes de iniciarse estas relaciones.

Palabras clave: Victimización; Perpetración; Violencia de género; Violencia en el noviazgo; Redes sociales.

Recibido: 04/05/2020 |Aprobado: 21/01/2021 |Publicado: 01/07/2021

c) (i)(-) Esta obra está bajo una Licencia Creative Commons Atribución-NoComercial-CompartirIgual 4.0
Internacional.

Financiación o proveniencia del artículo: Este artículo se deriva de la investigación doctoral titulada El Apoyo Social en la violencia de género en relaciones de pareja heterosexual. Caso Bogotá-Colombia y defendida en el año 2020 en la Facultad de Trabajo Social de la Universidad Complutense de Madrid.

¿Cómo citar este artículo? / How to quote this article?

Reina-Barreto, J. A. (2021). Victimización y perpetración de violencia en pareja adolescente y redes de apoyo en Colombia. Análisis con perspectiva de género. Prospectiva. Revista de Trabajo Social e intervención social, (32), 125150. doi: $10.25100 /$ prts.v0i32.9590. 
Reina-Barreto

\title{
Victimization and Perpetration of Violence in Adolescent Couples and Support Networks in Colombia. Analysis from a Gender Perspective
}

\begin{abstract}
This study analyses the victimization and perpetration of violence in youthful relations and other socio-demographic variables by comparing two groups, one that sought social support in circumstances of partner violence with another one that did not seek such support. It also describes the structure and density of social support networks based on a sample of 2,217 women between 13-19 years of age, who answered the Bogotá-2011 health macro-survey. Contingency tables with Chi-square contrast and Cramer's V coefficient were used to verify the intensity of the association among variables.

In victimization, $9 \%$ of the sample suffered violence by their partners, and of this sample $67.5 \%$ did not seek support. The group that sought support reported a high prevalence and intensity of violence suffered, with up to 19 different forms of violence reported by the same teenager. A higher prevalence and intensity of violence increased the seeking of support, although living and sharing with the aggressor decreased the search for support. In perpetration, $3.2 \%$ of the sample used violence against their male aggressor partner. The teens who reported the highest levels of violence did not perpetrate violence towards their aggressor partners in a major proportion. Regarding support networks, their close family was the main one reported. The findings suggest that young women experience gender-based violence in their relations, and male dominance continues to be present, invisible, and culturally legitimized in younger generations. The importance of design social policies and interventions that contribute to deconstructing unequal relationships before these relationships begin is evident.
\end{abstract}

Keywords: Victimization; Perpetration; Gender based violence; Dating Violence; Social networks.

Sumario: 1. Introducción, 2. Referente teórico y conceptual, 3. Metodología, 3.1 Muestra, 3.2 Instrumento, 3.3 Procedimiento, 4. Hallazgos, 4.1 Diferencias sociodemográficas según búsqueda de apoyo social, 4.2 Victimización. Sufrimiento de violencia de género según búsqueda de apoyo social, 4.3 Perpetración. Violencia hacia el agresor según búsqueda de apoyo social, 4.4 Redes de apoyo social: estructura y densidad, 4.5 Distinciones que sugieren violencia de género en relaciones de adolescentes en Colombia, 5. Conclusiones, 6. Referencias bibliográficas. 


\section{Introducción}

La violencia de género es una violación a los Derechos Humanos de las mujeres y su defensa se ha consagrado en tratados internacionales. En el caso colombiano, si bien el problema se encuentra incorporado a la legislación nacional desde 1996, (Cardona-Cuervo, Carrillo-Cruz y Caycedo-Guió, 2019) está en la agenda pública desde hace menos tiempo para garantizarse de manera real y efectiva (Hoyos y Benjumea, 2016; López-Rodríguez, 2019) y avanzar en el desarrollo social (Fondo de Población de las Naciones Unidas [UNFPA], 2019a, 2019b).

La mayor manifestación de violencia de género sucede en el ámbito de las relaciones de pareja, la ejerce la pareja o expareja masculina con o sin convivencia y se presenta desde la juventud según las estadísticas nacionales. De acuerdo con el Instituto Nacional de Medicina Legal y Ciencias Forenses de Colombia, entre 2009 y 2018 se registraron 522.453 peritajes por violencia entre cónyuges, siendo la víctima femenina en el 87,7\% de los casos y de ellas el 8,9\% era menor de 19 años (Acosta-Vélez, 2016, p. 296; Cifuentes-Osorio, 2018, p. 262; Lemos-Mena y Echeverri-Calero, 2019, p. 202; Marthé-Manjarres, 2017, p. 310; Pataquiva-Wilches, 2015, p. 139).

A la violencia que ejercen las parejas contra las mujeres adolescentes durante las primeras relaciones amorosas se la denomina con frecuencia violencia en el noviazgo (en inglés dating violence) y se la ha diferenciado de las relaciones amorosas adultas por la duración del vínculo, la no implicación de convivencia y el no tener compromisos económicos o un proyecto de familia. Se caracteriza, entre otras cuestiones, por el predominio de los términos perpetración y victimización para referirse, respectivamente, al ejercicio y sufrimiento de violencia (Gracia-Leiva, Puente-Martínez, Ubillos-Landa \& PáezRovira, 2019). Sin embargo, hay estudios mundiales de la OMS que muestran que se trata de un problema que afecta a las mujeres de manera sistemática durante todo su ciclo vital, que inicia en muchos casos en la adolescencia (World Health Organization, 2013), y que tiene varias denominaciones en castellano, como violencia machista, violencia contra la pareja (VCP del inglés intimate partner violence) (Lee, Pomeroy \& Bohman, 2007; McClennen, 2010) o violencia contra la mujer en la pareja (Jiménez-Rodrigo y Guzmán-Ordaz, 2015), y se refirieren, generalmente, a mujeres con experiencia de convivencia con el agresor. Otros autores matizan que la violencia de género se produce en relaciones de pareja con y sin convivencia y desde la adolescencia y juventud (Jennings et al., 2017; Reina-Barreto, 2020).

Respecto al estado de la cuestión acerca de la violencia en las primeras relaciones amorosas, un reciente meta-análisis sintetizaba los factores de protección y riesgo asociados a la violencia en el noviazgo. En los primeros se identificaron, entre otros, el apoyo social de pares y la amistad positiva. Entre los factores de riesgo se mencionaron: la edad (a partir de los 17 años y asociada a la violencia sexual), el embarazo precoz (asociado a abuso físico y 
sexual) o pertenecer a una minoría cultural. Otros trabajos sugerían una violencia mutua puesto que las mujeres perpetraban violencia física y los hombres violencia sexual, si bien las jóvenes eran mayoritariamente las víctimas de la violencia sexual (Gracia-Leiva et al., 2019).

Frente a los estudios que apuntan a una violencia mutua en las primeras relaciones erótico afectivas, otras investigadoras e investigadores interpelan si en aquellos análisis se han considerado los aportes de la teoría de género respecto a cómo se imbrican las relaciones dentro del orden social patriarcal, sus desigualdades y opresiones así como el modo en el que se impregnan en los comportamientos y los roles asumidos en la relación (Bosch-Fiol y Ferrer-Pérez, 2013; Heise, 1998; Lagarde, 2005, 2006; Mullender, 2000; Segato, 2003, 2016).

Así pues, y tras revisar la producción científica más relevante sobre el tema en Colombia, este artículo analiza, desde la perspectiva de género, el papel del apoyo social en la violencia sufrida por mujeres adolescentes a manos de su pareja (victimización) y en la violencia ejercida contra la pareja (perpetración), con el fin de contribuir a la comprensión de la violencia de género en las primeras relaciones de pareja. Se plantean cuatro objetivos específicos: 1) identificar la prevalencia de mujeres adolescentes que informan violencia de género en sus primeras relaciones de pareja (en adelante VGCP) y buscan apoyo social (en adelante AS); 2) establecer sí existen diferencias sociodemográficas significativas entre el grupo que busca AS por la VGCP sufrida respecto del grupo que no busca AS; 3) comparar las prevalencias en victimización y perpetración de violencia según búsqueda de AS; y 4) identificar la estructura y densidad de las redes de apoyo. Las hipótesis de trabajo son: En victimización, H1: A mayor prevalencia e intensidad de VGCP sufrida por las adolescentes más búsqueda de AS. H2: Convivir con el agresor desestimula la búsqueda femenina de AS. En perpetración, H3: las adolescentes ejercen menos violencia de la que sufren a manos de sus parejas y H4: A más violencia de género sufrida (victimización), más violencia ejercen las adolescentes contra su agresor (perpetración).

\section{Referente teórico y conceptual}

En Colombia, la denominación de la violencia de género en las relaciones de pareja aún no es homogénea y en este sentido se ha señalado recientemente la necesidad de delimitarla y definirla (Reina-Barreto, 2020). Así, el Instituto Nacional de Medicina Legal y Ciencias Forenses, en sus estadísticas sobre "violencia de pareja" incluye relaciones sin convivencia (novios o exnovios), con casos registrados desde los 10 años de edad (LemosMena y Echeverri-Calero, 2019, pp. 200-202), mientras que la macro-encuesta de salud, que también utiliza el término "violencia de pareja", aplica como filtros la experiencia de convivencia contabilizando los casos a partir de los 13 años de edad femenina (SánchezLara, 2015, p. 395). 
Reina-Barreto

Por lo anterior, se propone el término violencia de género en relaciones de pareja (VGCP) para referirse al amplio espectro de actos de violencia física, psicológica, sexual, verbal, económica, patrimonial, ciberviolencias, omisiones, amenazas, coerciones, control, acecho y privaciones de libertad en el marco de una relación de pareja heterosexual, indistintamente de que hubiera o no convivencia. Identifica como pareja al esposo, exesposo, pareja, expareja, compañero, excompañero, novio o exnovio. El término recoge además los aportes de Simón-Gil (2019) en cuanto a la secuela o daño social que produce la violencia de género y constituye en sí mismo una estrategia de visibilización, para deconstruir la producción y reproducción social de las invisibilidades del lenguaje patriarcal que encubren o disimulan este problema.

Por otra parte, en Colombia existe un creciente interés en el tema ante la evidencia empírica encontrada. Los estudios más recientes han comparado variables sociodemográficas, sociales o de violencia con muestras mixtas mayoritariamente. Se ha estudiado entre otros, la relación entre la exposición a la violencia de género en la familia de origen o en el entorno cercano y el perpetrar violencia en el noviazgo (Bonilla-Algovia y Rivas-Rivero, 2019a); el perpetrar o no violencia sexual contra su pareja (Rey-Anacona, 2017); el estudio de variables sociodemográficas como residir en zona rural o urbana o el papel de la edad en la violencia en el noviazgo (Redondo-Pacheco, Inglés-Saura y GarcíaLizarazo, 2017; Rey-Anacona, Martínez-Gómez y Londoño-Arredondo, 2017). Otros trabajos se han centrado en las representaciones sociales sobre la perpetración y la victimización (Jaramillo-Jaramillo, Holguín-Osorio y Ramírez-Ramírez, 2016); en los tipos e índices de violencia (Bernal-Pinilla, Hernández-Cuchumbe y Akl-Moanack, 2016), o en la vigencia de estereotipos de género, en actitudes de hostilidad, dominación, justificación, aceptación y naturalización de las primeras manifestaciones de violencia física (MontoyaRuiz, Cruz-Torrado y Leottau-Mercado, 2013). Sin embargo, no se ha encontrado evidencia relativa al estudio del apoyo social cuando la mujer ha sufrido violencia de género (victimización) y/o ejercido violencia contra su agresor (perpetración) en las primeras relaciones de pareja.

La evidencia encontrada demuestra la necesidad de abordar nuevas variables sociales desde análisis críticos, siendo la perspectiva de género el marco seleccionado para comprender cómo las condiciones socio-estructurales y socio-históricas determinan distinciones y diferencias en la VGCP (Camargo, 2019; Cleto, Covolan \& Signorelli, 2019; Ferrer-Pérez y Bosch-Fiol, 2019; Garzón-González, 2018; Jiménez-Rodrigo y GuzmánOrdaz, 2015; Reina-Barreto, 2020).

La teoría de género analiza cómo la estructura social patriarcal construye y sustenta el sistema de dominación masculina y subordinación femenina (Bourdieu, 2000), donde la relación de pareja interpreta bajo la misma jerarquía las relaciones de género. Las personas que conforman la pareja son a la vez productoras y reproductoras de modelos hetero- 
normativos estereotipados respecto al significado de ser mujer u hombre en sus hábitus (Bourdieu, 2000); por tanto encarnan y perpetúan las relaciones de desigualdad y de poder entre unos y otras (Lagarde, 2006; Segato 2003). Además, las prácticas socioculturales aceptadas para dicha relación minimizan, justifican, naturalizan y legitiman la violencia como una forma de relacionarse ya desde sus inicios (Garzón-González, 2018).

La VGCP responde a un proceso que inicia con la instauración de la dominación masculina en la relación, siendo unas de sus primeras estrategias el progresivo aislamiento social y familiar de la mujer y el debilitamiento de sus redes informales, las potenciales fuentes de AS. Varios estudios han identificado la capacidad mediadora del AS en el impacto negativo de la VGCP y en la elaboración de estrategias femeninas y alternativas vitales sin violencia (Guruge, Thomson, George \& Chaze, 2015; Lee et al., 2007; QueirogaSouto et al., 2016).

El AS es un constructo teórico de sumo interés para la investigación en y desde el Trabajo Social en tanto constituye uno de los ejes centrales del ejercicio profesional desde su fundación disciplinar (Capilla-Pérez y Villadóniga-Gómez, 2004; Lorente-Molina y Luxardo, 2018; Richmond, 2008; Zamanillo-Peral, 2018). Con relación a la VGCP, algunas investigaciones recientes han sugerido asignarle mayor protagonismo en la investigación del problema (Rodríguez-Fernández y Ortiz-Aguilar, 2018). Este estudio recoge dicha demanda y ubica al AS como variable dependiente para analizar la VGCP en las primeras relaciones de pareja en Colombia.

El AS es un metaconcepto multidimensional que ha sido definido de manera sintética como la "transacción de recursos o fuerzas derivados de las relaciones sociales en un determinado entorno social" (Lin, 1986). El AS suele analizarse desde tres perspectivas: estructural, funcional y contextual. La primera analiza, entre otros aspectos, la existencia previa de la red, su estructura y densidad. Las redes pueden ser natural-informal (familia, amistades, compañeras y compañeros de estudio o empleo) o formal-institucional (organizaciones comunitarias, ONG'S e instituciones del estado). La segunda perspectiva analiza los tipos específicos de apoyo, que son emocional, informacional, consejo y material o instrumental; así como la percepción de utilidad que del apoyo recibido, tiene quien demanda la ayuda. En la perspectiva contextual se analiza el contexto social en el que emergen estos procesos de apoyo, indagando acerca de las valoraciones socioculturales respecto de cuáles sucesos son susceptibles de ser objeto de ayuda (Barrera, 1986; Barrón-López, 1996; Caplan, 1974; Díaz, 1987; Gracia-Fuster, Herrero-Olaizola y Musitu-Ochoa, 1995; Gottlieb, 1985; House, 1981; Hombrados-Mendieta, 2013). En este trabajo únicamente se aborda la perspectiva estructural del AS. 


\section{Metodología}

Este estudio es de corte transversal y alcance descriptivo. Forma parte de una investigación doctoral acerca del apoyo social y la violencia de género en Colombia (ReinaBarreto, 2020). Toma como fuente de información secundaria la base de datos de la Encuesta Distrital de Demografía y Salud (en adelante EDDS-2011), realizada entre los meses de Junio de 2010 a mayo de 2011 por un total de 39 encuestadoras específicamente capacitadas en su aplicación. Es, hasta la fecha, la única encuesta realizada en la ciudad de Bogotá con una muestra representativa del territorio rural y urbano. La base de datos fue cedida por la Secretaría Distrital de la Mujer de Bogotá conforme a los protocolos de seguridad de la información y ética de la investigación. La EDDS-2011 está conformada por 10.186 casos de mujeres de entre 13 a 49 años, con una tasa de respuesta del 90,5\%.1

En cuanto al enfoque metodológico, la investigación se encuadra dentro del paradigma crítico feminista y entre sus opciones multi-metodológicas, en el empirismo feminista que plantea que los sujetos cognoscentes son quienes introducen sesgos en la actividad científica y en el método científico (Castañeda-Salgado, 2008, p. 56). Bajo esta perspectiva se analiza, además de los cuatro objetivos enunciados al final de la introducción, cuáles fueron los planteamientos con los que se concibe y operativiza la VGCP en el instrumento EDDS, así como posibles prácticas androcéntricas que afectasen el análisis y los resultados descriptivos publicados en el informe EDDS-2011. Este segundo aporte analítico, derivado de aplicar un método de investigación feminista, es posible gracias a la fuente de información secundaria de la que se sirve el estudio.

\subsection{Muestra}

La submuestra del estudio está compuesta por 2.217 mujeres de entre 13 a 19 años $(\overline{\boldsymbol{x}}$ $=16,1$ años, DE=1,9). El 55\% tenía a lo sumo 16 años, el 14,5\%, 15,6\% y 14,8\% tenían 17, 18 y 19 años respectivamente. El 91,2\% no tenía hijas(os). Según estado civil, el 91,2\% eran solteras, el 7,2\% convivían en unión de hecho, el 0,4\% estaban casadas y el 1,1\% separadas. Entre quienes convivían en pareja, el 72\% llevaba menos de dos años de convivencia, el $13,1 \%$ tres años y el 14,9\% entre $4-7$ años. El 60\% vivía en familias nucleares, el 39,2\% con familia extensa y el $0,8 \%$ en estructura unipersonal. Respecto al nivel educativo, el $82 \%$ informó haber realizado estudios secundarios, el 8,8\% universitarios, el 5,1\% técnicos y el 4,1\% primarios. El 62,2\% no tenía experiencia laboral, el 25,2\% informó tener un trabajo precario o estar en desempleo y el 12,5\% tenía trabajo formal. En auto-identificación étnica, el 2,3\% se auto-identificó como afrocolombiana y el 0,9\% perteneciente a una comunidad originaria.

1 El detalle del diseño muestral puede consultarse a partir de la página 523 en drive.google.com/file/d/1_XLolMyUMjwwGSxY0OFNU1m3D0kXGzJ8/view?usp=sharing. 


\subsection{Instrumento}

La EDDS-2011 aplicó el mismo cuestionario utilizado en la Encuesta Nacional de Demografía y Salud -ENDS- (en inglés Demographic Health Survey DHS) en los años 2000, 2005, 2010 y 2015, conformado por cinco formularios incluido el formulario individual. ${ }^{2}$ Éste recoge información de variables sociodemográficas, económicas, laborales y de maternidad (módulos 1-2-6-8) y el módulo denominado violencia doméstica indaga sobre la VGCP en mujeres con experiencia de convivencia en pareja siempre que se confirme la privacidad. Utiliza la escala de tácticas de conflictos modificada o CTS-2 (Straus, Hamby, McCoy \& Sugarman, 1996), usada a nivel mundial para medir este tipo de violencia (Bonilla-Algovia y Rivas-Rivero, 2019a; Camargo, 2019; Jennings et al., 2017). El módulo también pregunta acerca de la violencia contra la mujer ejercida por un novio, exnovio o expareja; la violencia ejercida por la mujer hacia la pareja (preguntas Q1114 Q1115 Q1116); la búsqueda de AS y las redes de apoyo informales y/o formales (preguntas Q1149 y Q1150).

\subsection{Procedimiento}

Con el fin de contrastar la extemporaneidad de los datos, se procedió a comparar la variación porcentual en las variables de interés EDDS-2011 respecto los últimos informes nacionales ENDS 2010-2015 (fuente de información con capacidad de evidenciar cierta trazabilidad del fenómeno). Se confirmó que la prevalencia de los datos EDDS-2011 no refleja cambios significativos respecto ambos informes ENDS. Por lo tanto la fuente de información seleccionada continúa aportando información relevante para el estudio.

Seguidamente se revisaron exhaustivamente las preguntas y opciones de respuesta del cuestionario individual para capturar toda manifestación de violencia cometida por una pareja. Se identificaron en total 36 indicadores de violencia de la pareja contra la mujer. Con estos indicadores se creó una variable que contabilizó cuántos actos distintos de violencia informaba una misma mujer con el fin de servir de indicador de intensidad de la VGCP. Las tipologías de violencia verbal, física, económica, sexual, de control y amenazas, fueron recodificadas para agrupar sus indicadores respectivos. Se creó una variable dicotómica de VGCP. Las variables número de hijas(os), nivel educativo y situación laboral femenina fueron recodificadas para una mejor implementación de las técnicas elegidas para el análisis estadístico de la información. Por otra parte y para aprovechar la riqueza de información de variables multirespuesta tabuladas en la matriz de datos EDDS-2011 como variables tipo cadena (limitando de manera importante el análisis) se utilizó el método de algoritmos de sintaxis para desagregar las 22 posibles fuentes de apoyo (que conformarían la red de apoyo

2 Cuestionario 
formal o informal) y las tres formas de violencia que ejerce la mujer: física, control y verbal. El detalle del procedimiento se puede consultar en Reina-Barreto (2020).

Cabe señalar que, debido a la estructura de preguntas filtro del cuestionario, únicamente se recogió información de variables tipo cadena cuando las mujeres informaron experiencia de convivencia en pareja $(n=158)$.

Posteriormente se comparó al grupo que buscó AS por la VGCP con respecto del grupo que no buscó AS en cuanto a características sociodemográficas, victimización, perpetración y pensamientos de separación por la violencia sufrida (en mujeres que convivían en pareja). Finalmente se identificaron las fuentes de apoyo en las redes formal e informal y su densidad.

Respecto a las técnicas de análisis, se realizaron tablas de contingencia con contraste de independencia Chi-cuadrado de Pearson y un nivel de confianza del 95\% (p valor<0.05). También se utilizó el coeficiente V de Cramer para comprobar la intensidad de la relación entre variables, considerándola significativa cuando fuera mayor de 0,3 conforme a las consideraciones de la prueba para las ciencias sociales. Para el análisis de los datos se utilizó SPSS-23.

\section{Hallazgos}

Las mujeres adolescentes sufrieron mucha más violencia por parte de sus parejas de la que ellas ejercieron hacia sus agresores y la búsqueda femenina de apoyo fue mayor cuanta más violencia habían experimentado, resultando dicha relación estadísticamente significativa. En Victimización, el 9\% de la muestra sufrió VGCP en sus relaciones de pareja $(n=200)$ y en el $71 \%$ de los casos, sus parejas masculinas perpetraron violencia contra ellas el año anterior a la encuesta. Sin embargo, sólo el 32,5\% buscó apoyo, por lo tanto el 67,5\% de mujeres adolescentes sufrió la violencia de género en solitario y sin buscar ningún apoyo.

El 96,8\% de la muestra de mujeres adolescentes no ejerció ninguna forma de violencia contra su pareja masculina $(n=2.146)$ y el3,9\% si lo hizo $(n=71)$; concretamente el 2,6\% ejerció control y las violencias verbal y física obtuvieron prevalencias residuales $(0,5 \%$ y $0,1 \%$ respectivamente), sin que dicha relación resultara estadísticamente significativa. De las adolescentes que ejercieron alguna violencia contra su pareja $(n=71)$ prácticamente todas lo hicieron contra parejas masculinas que las habían violentado $(n=68)$. De la muestra $(n=2.217)$ sólo tres mujeres adolescentes informaron ejercicio de violencia de tipo control sin sufrir VGCP.

Los análisis que se presentan a continuación comparan el subgrupo de mujeres adolescentes que informaron VGCP y buscaron AS por esta violencia $(n=65)$, (en adelante 
Reina-Barreto

grupo Si-AS) con el subgrupo que también informó VGCP pero no buscó apoyo (n=135) (en adelante grupo No-AS) en cuanto a características sociodemográficas, victimización y perpetración de violencia.

\subsection{Diferencias sociodemográficas según la búsqueda de apoyo social}

Al comparar las prevalencias totales de cada variable sociodemográfica según la búsqueda de AS (Tabla 1), en el grupo No-AS se encontró que eran mayoritariamente adolescentes menores de 17 años, eran madres que convivían con el agresor en familias nucleares con cabeza de hogar masculina. No contaban con experiencia laboral, estaban desempleadas o informaron de empleos precarios y sus niveles educativos eran inferiores respecto a los informados por el grupo Si-AS. De acuerdo con el estrato socioeconómico, siendo que la mayoría de la submuestra se ubicó en niveles bajos $(<2=73 \%)$ las adolescentes del grupo No-AS estuvieron más representadas tanto en los estratos inferiores $(<2=75,5 \%)$ como en los estratos superiores (total submuestra estrato entre 4- $6=3 \%$ vs. estrato entre 4- 6 para No-AS=3,7\%). Según auto-identificación étnica, todas aquellas que se reconocieron procedentes de comunidades originarias no buscaron apoyo (3,7\%), así como las afrocolombianas, que también quedaron más representadas en este grupo (total submuestra afrocolombianas $=3,5 \%$ vs. afrocolombianas No-AS=4,4\%).

Por otra parte, en el grupo Si-AS la mayoría eran adolescentes mayores de 18 años, solteras o separadas, aunque también en uniones libres y con estudios técnicos o universitarios. Agrupó a más adolescentes sin hijas(os) que vivían en familias extensas con cabeza de hogar femenina. Un porcentaje algo mayor contaba con empleo formal (total submuestra empleo formal $=23 \%$ vs. empleo formal para $\mathrm{Si}-\mathrm{AS}=27,7 \%$ ). Respecto al estrato socioeconómico, se ubicaron más en estrato medio (total submuestra estrato 3=18\% vs. estrato 3 para $\mathrm{Si}-\mathrm{AS}=21,5 \%)$. Y en relación con la autoidentificación étnica $-\mathrm{y}$ como se deduce del párrafo anterior- ninguna adolescente de una comunidad originaria buscó AS por la VGCP sufrida (Tabla 1).

De las diez variables sociodemográficas femeninas analizadas (Tabla 1) resultaron estadísticamente significativas el estado civil y la cabeza del hogar con la búsqueda de AS por la VGCP, aunque la magnitud de asociación fue baja. 
Reina-Barreto

Tabla 1. Diferencias sociodemográficas mujeres adolescentes que informaron violencia de género en sus relaciones de pareja *búsqueda de apoyo social. En \%.

\begin{tabular}{|c|c|c|c|c|}
\hline \multicolumn{2}{|c|}{ Característica sociodemográfica } & \multicolumn{2}{|c|}{ Búsqueda de apoyo social } & \multirow{2}{*}{ Total $(n=200)$} \\
\hline & & No $(n=135)$ & $\mathrm{Si}(\mathrm{n}=65)$ & \\
\hline \multirow[t]{5}{*}{ Edad de la mujer } & $13-16$ a. & $16,9 \%$ & $12,3 \%$ & $16 \%$ \\
\hline & $17 \mathrm{a}$. & $22,2 \%$ & $16,9 \%$ & $20,5 \%$ \\
\hline & 18 a. & $30,4 \%$ & $33,8 \%$ & $31,5 \%$ \\
\hline & 19 a. & $30,4 \%$ & $36,9 \%$ & $32,5 \%$ \\
\hline & & $100 \%$ & $100 \%$ & $100 \%$ \\
\hline \multirow[t]{5}{*}{ Estado civil* } & Soltera & $14,8 \%$ & $33,8 \%$ & $21 \%$ \\
\hline & Casada & $3,7 \%$ & $4,6 \%$ & $4 \%$ \\
\hline & Unión de hecho & $70,4 \%$ & $47,7 \%$ & $63 \%$ \\
\hline & Separada & $11,1 \%$ & $13,8 \%$ & $12 \%$ \\
\hline & & $100 \%$ & $100 \%$ & $100 \%$ \\
\hline \multirow{5}{*}{$\begin{array}{l}\text { Años de convivencia con la pareja } \\
\text { (mujeres con convivencia actual) }\end{array}$} & NS / NR & & $2,9 \%$ & $0,7 \%$ \\
\hline & Hasta 2 años & $65 \%$ & $76,5 \%$ & $68 \%$ \\
\hline & 3 años & $17 \%$ & $9 \%$ & $15 \%$ \\
\hline & 4 a 7 años & $18 \%$ & $11,7 \%$ & $16,4 \%$ \\
\hline & & $100 \%$ & $100 \%$ & $100 \%$ \\
\hline \multirow[t]{4}{*}{ Número de hijas(os) } & Sin hijas(os) & $45,2 \%$ & $50,8 \%$ & $47 \%$ \\
\hline & Entre 1 y 2 & $53,3 \%$ & $49,2 \%$ & $52 \%$ \\
\hline & 3 o más & $1,5 \%$ & & $1 \%$ \\
\hline & & $100 \%$ & $100 \%$ & $100 \%$ \\
\hline \multirow[t]{5}{*}{ Nivel educativo } & Primaria o menor & $11,1 \%$ & $3,1 \%$ & $8,5 \%$ \\
\hline & Secundaria & $78,5 \%$ & $81,5 \%$ & $79,5 \%$ \\
\hline & Técnico & $5,9 \%$ & $9,2 \%$ & $7 \%$ \\
\hline & Universitario & $4,4 \%$ & $6,2 \%$ & $5 \%$ \\
\hline & & $100 \%$ & $100 \%$ & $100 \%$ \\
\hline \multirow[t]{5}{*}{ Situación laboral } & Sin experiencia & $28,1 \%$ & $24,6 \%$ & $27 \%$ \\
\hline & Empleo precario/ & & & \\
\hline & desempleada & $51,1 \%$ & $47,7 \%$ & $50 \%$ \\
\hline & Empleo formal & $20,7 \%$ & $27,7 \%$ & $23 \%$ \\
\hline & & $100 \%$ & $100 \%$ & $100 \%$ \\
\hline \multirow[t]{4}{*}{ Formas de convivencia en familia } & Unipersonal & & $3,1 \%$ & $1 \%$ \\
\hline & Nuclear & $49,6 \%$ & $40 \%$ & $46,5 \%$ \\
\hline & Extensa & $50,4 \%$ & $56,9 \%$ & $52,5 \%$ \\
\hline & & $100 \%$ & $100 \%$ & $100 \%$ \\
\hline \multirow[t]{4}{*}{ Cabeza del hogar* } & Femenina & $2,2 \%$ & $10,8 \%$ & $5 \%$ \\
\hline & Masculina & $40,0 \%$ & $27,7 \%$ & $36 \%$ \\
\hline & NS / NR & $57,8 \%$ & $61,5 \%$ & $59 \%$ \\
\hline & & $100 \%$ & $100 \%$ & $100 \%$ \\
\hline \multirow[t]{4}{*}{ Auto-identificación étnica } & Ninguna & $91,9 \%$ & $98,5 \%$ & $94 \%$ \\
\hline & Comunidad originaria & $3,7 \%$ & & $2,5 \%$ \\
\hline & Afrocolombiana & $4,4 \%$ & $1,5 \%$ & $3,5 \%$ \\
\hline & & $100 \%$ & $100 \%$ & $100 \%$ \\
\hline \multirow[t]{5}{*}{ Estrato socioeconómico } & Estrato $<2$ & $75,5 \%$ & $67,7 \%$ & $73 \%$ \\
\hline & Estrato 3 & $16,3 \%$ & $21,5 \%$ & $18,0 \%$ \\
\hline & Entre 4-6 & $3,7 \%$ & $1,5 \%$ & $3 \%$ \\
\hline & NS / NR & $4,4 \%$ & $9,2 \%$ & $6 \%$ \\
\hline & & $100 \%$ & $100 \%$ & $100 \%$ \\
\hline
\end{tabular}


Reina-Barreto

\subsection{Victimización. Sufrimiento de violencia de género según búsqueda de apoyo social}

Tanto en el grupo No-AS como en Si-AS las mujeres adolescentes informaron altas prevalencias de violencia cometida por sus parejas de tipo física, sexual, verbal, económica, control, y amenaza, siendo mayores las prevalencias informadas por el grupo Si-AS, que alcanzaron hasta el $80 \%$ en violencia física (Figura 1). En este grupo las violencias física y sexual registraron el doble y triple de prevalencia respecto las del grupo No-AS (80\% vs. $39,3 \%$ y $9,2 \%$ vs. $3 \%$ respectivamente), siendo similares en violencia económica (Si$\mathrm{AS}=27,7 \%$ vs. No-AS=28,1\%). La violencia física que perpetró la pareja masculina contra la mujer adolescente se asoció con la búsqueda femenina de apoyo social con una magnitud alta; y la violencia sexual, el control y las amenazas resultaron estadísticamente significativas aunque con una magnitud de asociación baja.

Figura 1. Victimización. Tipologías de violencia de género informada por mujeres adolescentes en sus relaciones de pareja *búsqueda de apoyo social. En \%.

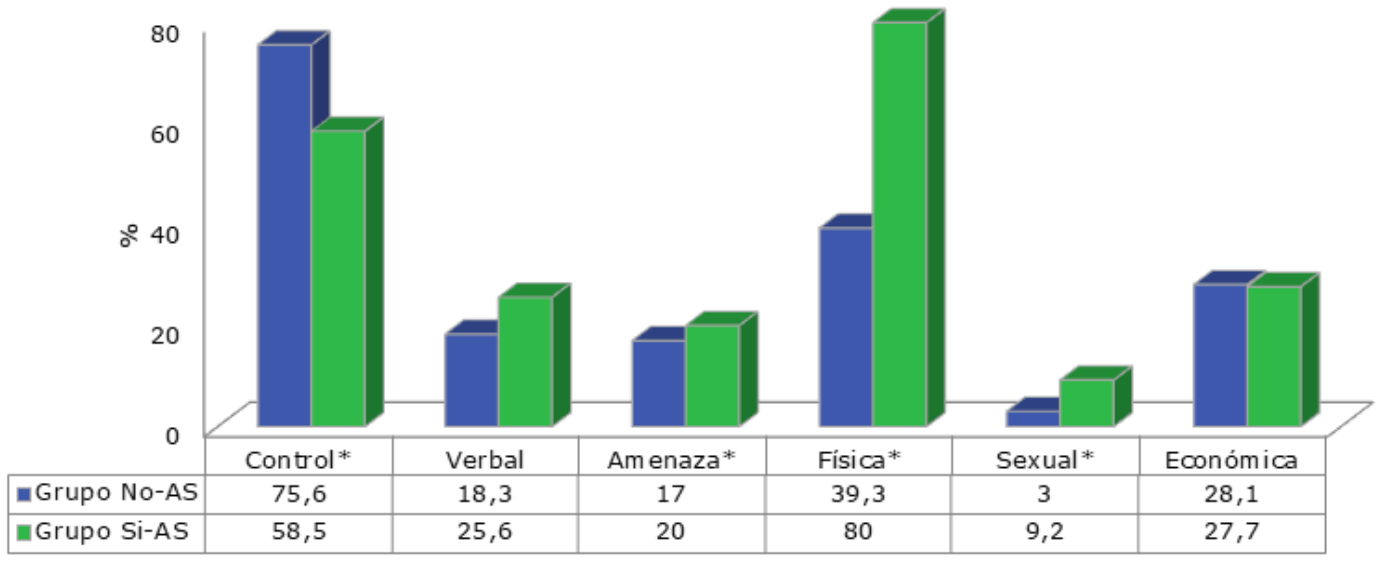

Fuente: elaboración propia. 2021

Al comparar los actos de violencia perpetrados por la pareja agresora que informó una misma adolescente, se encontró que el grupo Si-AS acumuló hasta 19 formas distintas de $\operatorname{VGCP}(\overline{\boldsymbol{X}}=4,6 \mathrm{DE}=4,8 \mathrm{Me}=3)$, mientras el grupo No-AS acumuló hasta 13 formas $(\overline{\boldsymbol{x}}=3,5$ $\mathrm{DE}=3,02 \mathrm{Me}=3)$, demostrando que ambos grupos de mujeres adolescentes soportaron violencias de sus parejas combinadas de muchas formas, aunque quienes buscaron apoyo sufrieron más violencia acumulada (Figura 2). 
Reina-Barreto

Figura 2. Victimización. Intensidad de la violencia de género informada por mujeres adolescentes en sus relaciones de pareja *búsqueda de apoyo social. En \%.

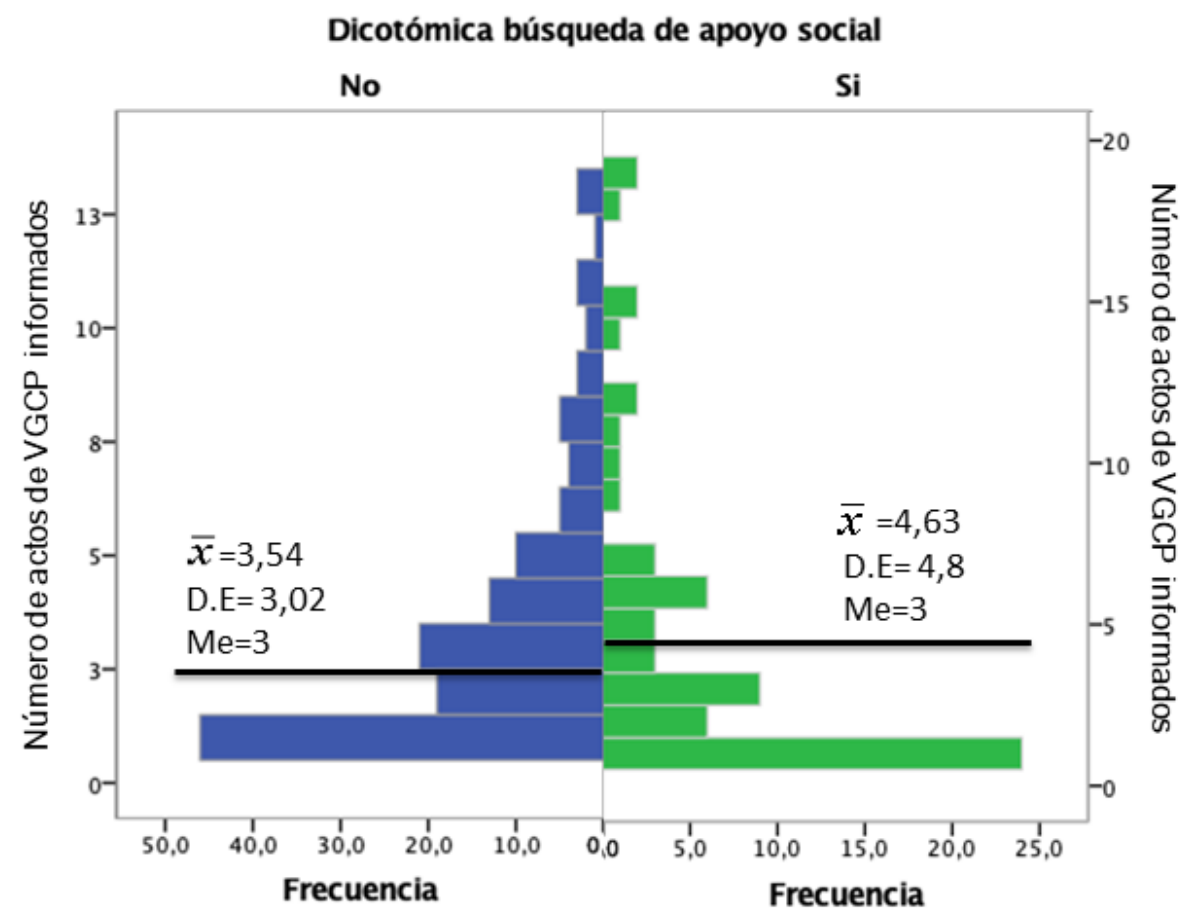

Fuente: elaboración propia. 2021.

Al detenerse en los casos de las adolescentes que convivían con el agresor para indagar el inicio de la violencia perpetrada contra ellas ( $n=134)$, el 69\% informó que la misma comenzó antes de cumplir un año de convivencia en pareja y el 5,5\% durante el noviazgo. El 33,1\% había pensado en separarse. El grupo Si-AS asoció el 77\% de las razones a la violencia de género que perpetraba la pareja contra ella. Así la violencia física, psicológica y/o sexual fue la causa principal informada (46,2\%), seguida de la violencia económica, expresada como incumplimiento de los deberes de esposo/padre $(30,8 \%)$. En el grupo NoAS las razones asociadas a la VGCP supusieron un 37,9\%, de las cuales el 20,7\% refirió la violencia física psicológica y/o sexual, el 10,3\% la violencia psicológica manifestada como infidelidad masculina y el 6,9\% por la violencia económica (Tabla 2). El contraste Chicuadrado reveló que el tener pensamientos de separación por la VGCP se asoció a la búsqueda de AS, si bien la magnitud fue baja. 
Reina-Barreto

Tabla 2. Victimización. Mujeres adolescentes. Pensamientos de separación por la violencia de género en relación de pareja *búsqueda de apoyo social. En \%.

\% dentro de Dicotómica búsqueda de apoyo social.

\begin{tabular}{|c|c|c|c|c|}
\hline & & \multicolumn{2}{|c|}{ Búsqueda de apoyo social } & \multirow{2}{*}{ Total } \\
\hline & & No & $\mathrm{Si}$ & \\
\hline \multirow{4}{*}{$\begin{array}{l}\text { Razón para pensar en } \\
\text { separarse de la pareja } \\
\text { con quien convive* }\end{array}$} & $\begin{array}{c}\text { Violencia del cónyuge } \\
\text { (física/psicológica/sexual) }\end{array}$ & $20,7 \%$ & $46,2 \%$ & $28,6 \%$ \\
\hline & $\begin{array}{l}\text { Violencia psicológica: } \\
\text { Infidelidad cónyuge }\end{array}$ & $10,3 \%$ & $0 \%$ & $7,1 \%$ \\
\hline & $\begin{array}{l}\text { Violencia económica: } \\
\text { incumplimiento deberes } \\
\text { esposo/padre }\end{array}$ & $6,9 \%$ & $30,8 \%$ & $14,3 \%$ \\
\hline & Otras razones & $62,1 \%$ & $23 \%$ & $49,9 \%$ \\
\hline Total & & $100 \%$ & $100 \%$ & $100 \%$ \\
\hline
\end{tabular}

\subsection{Perpetración. Violencia hacia el agresor según búsqueda de apoyo social}

Como ya se anticipó, el 96,8\% de la muestra de mujeres adolescentes no ejerció ninguna violencia hacia su pareja $(n=2.146)$, siendo por tanto el 3,9\% quienes sí la ejercieron alguna vez $(n=71)$ y de ellas prácticamente la totalidad habían sufrido violencia de sus parejas $(n=68)$. El 2,8\% lo hicieron durante el año anterior a la encuesta $(n=63)$. Las tipologías de violencia informadas por las mujeres fueron, por este orden, el control $(2,6 \%)$, la violencia verbal $(0,4 \%)$ y la violencia física $(0,09 \%)$. De la muestra de mujeres adolescentes $(n=2.217)$ tres ejercieron violencia de tipo control sin sufrir $\operatorname{VGCP}(0,1 \%)$.

Con el fin de analizar con mayor profundidad la dinámica de victimización y perpetración de violencia según la búsqueda de apoyo social, se compararon nuevamente los mismos datos para el subgrupo de mujeres que informaron VGCP $(n=200)$ comparando el grupo Si-AS $(n=65)$ con el grupo No-AS $(n=135)$, de ahí que las prevalencias señaladas anteriormente en el segundo párrafo de hallazgos varíen.

Se encontró que las adolescentes del grupo Si-AS mayoritariamente no ejercieron ninguna violencia hacia la pareja agresora (73,8\% vs. total subgrupo adolescentes con VGCP que nunca han ejercido violencia hacia el agresor=66\%). Además informaron menos violencia de tipo control y violencia verbal respecto del total registrado para todas las adolescentes con experiencia de VGCP (21,5\% y 3,1\% vs. $28 \%$ y $5 \%$ respectivamente). Aunque la prevalencia de violencia física ejercida por la mujer hacia la pareja agresora fue 
Reina-Barreto

residual (1\%), la prevalencia fue mayor en el grupo Si-AS (1,5\%) (Tabla 3). La relación entre ejercicio de violencia de las adolescentes hacia la pareja agresora y la búsqueda de apoyo social no resultó estadísticamente significativa.

Tabla 3. Perpetración. Mujeres adolescentes que informaron violencia de género en sus relaciones de pareja y han ejercido violencia hacia el agresor *búsqueda de apoyo social. En \%.

\begin{tabular}{ccccc} 
\% dentro de Dicotómica búsqueda de apoyo social & & & \\
\hline & & \multicolumn{2}{c}{ Búsqueda de apoyo social } & \multirow{2}{*}{ Total } \\
\cline { 3 - 4 } & & No & Si & \\
\hline \multirow{2}{*}{$\begin{array}{c}\text { Tipos de violencia } \\
\text { perpetrada }\end{array}$} & Nunca lo ha violentado & $62,2 \%$ & $73,8 \%$ & $66 \%$ \\
& Control & $31,1 \%$ & $21,5 \%$ & $28 \%$ \\
& Verbal & $5,9 \%$ & $3,1 \%$ & $5 \%$ \\
& Física & $0,7 \%$ & $1,5 \%$ & $1 \%$ \\
\end{tabular}

Fuente: elaboración propia. 2021

\subsection{Redes de apoyo social: estructura y densidad}

Se ha mencionado que sólo el 32,5\% de mujeres adolescentes buscó apoyo social por victimización en VCGP. De ellas, el 60\% acudió a su red informal de apoyo, el 11\% a la red formal y el $29 \%$ a ambas redes.

En la red informal las fuentes de apoyo citadas fueron en este orden: la familia de origen de la adolescente (65,5\% acudieron a su madre-padre, 25,9\% a otros familiares y 3,4\% a su madrastra-padrastro), el grupo de pares $(12,1 \%)$, la familia del agresor $(5,2 \%)$ y personas de otros entornos (1,2 \% a su docente, empleadora o empleador). En lo relativo a la densidad de la red informal, el $83 \%$ acudieron a una única persona y el $17 \%$ a dos personas.

En cuanto a la red formal, las instituciones a donde acudieron fueron en este orden: la fiscalía $(46,2 \%)$, la comisaría de familia $(26,9 \%)$, la policía $(23 \%)$ y otras instituciones no identificadas (7,7\%). El 88,5\% acudió a una sola institución y el 11,5 \% a dos entidades.

El contraste estadístico reveló significancia entre ambas redes de apoyo, lo que indicaría que la búsqueda de AS en la red informal se relacionó con la búsqueda de AS institucional. 


\subsection{Distinciones que sugieren violencia de género en relaciones de adolescentes en Colombia}

Este trabajo se plantea cuatro objetivos específicos: 1) identificar la prevalencia de mujeres adolescentes que informan violencia de género en sus primeras relaciones de pareja (VGCP) y buscan apoyo social (AS); 2) establecer sí existen diferencias sociodemográficas significativas entre el grupo que busca AS por la VGCP sufrida respecto del grupo que no busca AS; 3) comparar las prevalencias en victimización y perpetración de violencia según búsqueda de AS; y 4) identificar la estructura y densidad de las redes de apoyo. Las hipótesis de trabajo son: En victimización, H1: A mayor prevalencia e intensidad de VGCP sufrida por las adolescentes más búsqueda de AS. H2: Convivir con el agresor desestimula la búsqueda femenina de AS. En perpetración, H3: las adolescentes ejercen menos violencia de la que sufren a manos de sus parejas y H4: A más violencia de género sufrida (victimización), más violencia ejercen las adolescentes contra su agresor (perpetración).

El conjunto de resultados sugiere que la violencia de género está presente desde la génesis de las relaciones de pareja de las mujeres adolescentes de Bogotá en casi uno de cada diez casos (9\%). Los hallazgos presentan tres distinciones para el contexto colombiano que refuerzan la premisa de que se trata de violencia de género y no de violencia mutua o bidireccional como se entienden y utilizan los descriptores victimización y perpetración cuando se estudia la violencia en las relaciones de adolescentes. Primera, la mayoría de las adolescentes informa múltiples formas de violencia machista acumuladas (Figuras 1 y 2) que sufren sin buscar ningún apoyo $(67,5 \%$ ). Quienes buscan apoyo sufrieron todavía mayor intensidad de violencia, encontrándose una asociación estadística entre buscar el apoyo en la red informal y buscarlo también en la red formal, aportando evidencia empírica para aceptar la primera hipótesis (H1).

Segunda, no buscar apoyo se asocia con la convivencia con el agresor y con que él sea reconocido como la cabeza del hogar (Tabla 1), aportando evidencia empírica para aceptar la segunda hipótesis (H2). Cabe resaltar que a pesar de la corta edad de las mujeres de la muestra (máximo 19 años), dos de cada tres conviven con el agresor (67\%) aun cuando la violencia comenzó antes del año de convivencia (69\%), tal vez influenciadas por el hecho de que ya son madres $(53 \%)$.

Tercera, la comparación de los datos relativos a victimización y perpetración (Figuras 1 y 2 y Tabla 3) indicarían que la violencia que ejerce la pareja contra la mujer adolescente constituye un instrumento de opresión que opera especialmente mediante el control y la violencia física para establecer y afianzar la dominación masculina en la relación, que se encubre como muestras de amor romántico; mientras que las mujeres adolescentes mayoritariamente no ejercen violencia hacia sus parejas agresoras, siendo además muy distintas las prevalencias de violencias registradas en cada caso. Así pues, los hallazgos 
aportan evidencia empírica para aceptar la tercera hipótesis planteada $(\mathrm{H} 3)$ y rechazar la cuarta (H4).

Deteniéndose en el hallazgo de que la mayor prevalencia de violencia femenina hacia su pareja agresora es el control (2,6\%), este dato encuentra una explicación diferenciada para el contexto colombiano donde todavía se encuentra fuertemente arraigada y normalizada la permisividad social hacia prácticas de infidelidad masculina documentadas desde la época colonial (Pachón, 2007; Ramírez-Rodríguez, 2000) y en la que la mujer asume una actitud de vigilancia acerca de los comportamientos de su pareja intentando evitar o prevenir que dicha infidelidad se geste. Por lo tanto, el control femenino podría constituir una estrategia de autoprotección frente a este tipo de violencia psicológica. Adicionalmente, dado que la mayoría de las adolescentes con VGCP convivían con el agresor, el control también podría explicarse como una forma de evitar el derroche de los recursos familiares, puesto que otra práctica social extendida es el consumo de alcohol. Finalmente los actos de control femenino se encubren como muestras de amor tal y como ocurre con el control que ejerce el hombre contra la mujer. Por otra parte y con respecto a la prevalencia de la violencia física femenina hacia la pareja agresora, aunque residual (Tabla 3), Heise (1998) explica que en contextos socioculturales patriarcales, como el que aquí se estudia, la violencia constituye un mecanismo de defensa activa contra la VGCP.

Al contrastar estos resultados con otros estudios, se encontró que la menor búsqueda de AS por la VGCP se registró en las adolescentes con mayores niveles de control masculino y sugiere que afrontan solas la violencia. Coincide con el estudio de Jennings et al. (2017) quienes indicaban que el aislamiento femenino reducía la probabilidad de buscar apoyos. En Brasil y México identificaban que el aislamiento social se ejercía como método de control y represión masculina, por vergüenza y/o por miedo femenino a nuevos episodios de violencia (Albuquerque-Netto, Vasconcelos-Moura, Azevedo-Queiroz, Costa-Leite \& Fernandes e Silva, 2017; Vázquez-García y Castro, 2011). En Colombia se sugería que este aislamiento constituía una forma más de victimización (Redondo-Pacheco et al., 2017).

Puesto que el aislamiento femenino parece relacionarse con el control masculino, tal condición sugiere que las adolescentes tienen dificultades para solicitar apoyo a su red social pero también que favorece la permanencia en la relación. Al respecto, en Colombia, algunas investigadoras señalaban que el control se asocia a la justificación y/o minimización de la VGCP y a la vigencia del mito del amor romántico que interpreta sus manifestaciones como muestras de amor, tal y como este estudio también ha señalado, facilita el dominio masculino. Y sería más fácil cuanto más aislada se encuentre una mujer de su entorno social. Instaurada la dominación masculina, disminuye la necesidad de utilizar violencia física contra ella -el tipo de violencia más identificada socialmente como tal (Ariza-Sosa 2013; Reina-Barreto, 2016). 
En cuanto a las características sociodemográficas significativas de este estudio (adolescentes que conviven con el agresor en hogares con cabeza masculina son quienes menos apoyo buscan), indican la vigencia del estereotipo de familia patriarcal, coincidiendo con un estudio de Bolivia donde se encontró que la probabilidad de ejercer VGCP era mayor en este tipo de hogares (Camargo, 2019). Quienes más apoyo buscaron fueron las solteras o separadas y en hogares con cabeza femenina, lo que sugiere que, al no estar expuestas al dominio masculino de la misma manera que aquellas que viven con el agresor, se encontrarían menos aisladas de sus redes primarias de apoyo.

Por otra parte los hallazgos relativos a violencia sufrida (victimización) resultaron significativos con búsqueda de AS, coincidiendo con otras investigaciones de Colombia, México o Norteamérica donde las mujeres jóvenes informaron más violencia que sus parejas (Bonilla-Algovia y Rivas-Rivero, 2019b; Jaramillo-Jaramillo et al., 2016; Jennings et al., 2017; Medina-Núñez y Medina-Villegas, 2019; Montoya-Ruiz et al., 2013), pero difieren de otros estudios latinoamericanos donde no emergieron diferencias significativas en victimización y perpetración (Rey-Anacona et al., 2017; Vivanco-Muñoz, Espinoza-Moraga, RomoTregear, Véliz-Burgos y Vargas-Peña, 2015) o donde se informa violencia bidireccional (Espinoza-Moraga, Vivanco-Muñoz, Veliz-Burgos y Vargas-Peña, 2019).

Otra explicación a la alta prevalencia de victimización sin buscar apoyo (Figura 2), como ya se dijo, se encuentra en la naturalización de muchos actos de violencia incorporados como códigos del amor romántico, restándosele importancia, así la adolescente no percibe ni el peligro ni el incremento de la frecuencia e intensidad de la violencia. Este hallazgo coincide con lo planteado por Pataquiva-Wilches (2015) en Colombia, quien señala que únicamente la violencia física que genera daños se identifica en el imaginario social como violencia en pareja. Otra investigación brasileña señalaba que sólo cuando la violencia revestía gravedad y afectaba a las hijas(os) las jóvenes buscan AS (Baragatti, Carlos, Cruz-Leitão, Carvalho-Ferriani \& Silva, 2018). En la misma línea, varios estudios internacionales apuntaban resultados similares (Jaramillo-Jaramillo et al., 2016; Montoya-Ruiz et al., 2013; Pereira-Gomes, Freire-Diniz, Araújo dos Reis \& LorenziniErdmann, 2015).

En lo relativo a victimización, a excepción del control, todas las demás violencias fueron exacerbadas en el grupo que busca AS (Figuras 1 y 2), siendo tres veces más la violencia sexual sufrida. En este sentido, llama la atención la altísima prevalencia de madres en la muestra, lo que lleva a preguntarse si fue una decisión consensuada de pareja o es en cambio, un efecto de la violencia sexual encubierta e invisibilizada en la relación, tal y como han identificado otros estudios internacionales (Reina-Barreto, Criollo-Espín y FernándezD'Andrea, 2019; Vázquez-García y Castro, 2011). Otro reciente metaanálisis señala que la violencia sexual está fuertemente asociada a las mujeres a partir de los 17 años en las primeras relaciones de pareja (Gracia-Leiva et al., 2019). 
Finalmente y en cuanto al AS, este estudio identifica que dos de cada tres adolescentes no buscan AS cuando sufren VGCP $(67,5 \%)$ y cuando lo hacen, acuden a la red informal, sobre todo a su familia de origen y/o al grupo de pares. La red formal fue mucho menos informada, refiriéndose la mayoría de las ocasiones a la fiscalía o la comisaría de familia. Ambas redes se asociaron significativamente. Hallazgos similares se encontraron en Brasil. Pereira-Gomes et al. (2015) identificaron a la familia y las amistades como las fuentes de apoyo más informadas por jóvenes violentadas por sus parejas; Baragatti et al. (2018) puntualizaban que la línea de apoyos se daba entre mujeres. Albuquerque-Netto, Vasconcelos-Moura, França-Araujo, Nascimento-Souza \& Fernandes e Silva (2017) señalaron que la densidad de la red formal era mayor a la de la red informal, siendo por tanto inversos a los hallazgos aquí presentados. El mismo estudio encontró que la violencia influía en la selección femenina de fuentes de apoyo y que cuando no contaban con apoyo familiar recurrían solas a la red formal. Otros estudios revelaban que no contar con apoyo familiar dificultaba el proceso de romper el ciclo de violencia, además de contribuir a su normalización y naturalización (Brock-Carneiro et al., 2019; Pereira-Gomes et al., 2015), lo que podría estar relacionado para el contexto sociocultural colombiano, con el hecho de que pocas adolescentes busquen apoyo por la VGCP como aquí se ha demostrado.

Finalmente, el estudio presenta algunas limitaciones. Utilizar una fuente secundaria no permitió analizar otras dimensiones del AS estructural si bien se alcanzaron los objetivos propuestos. Así mismo convendría profundizar estos resultados con enfoque cualitativo y ampliarlo a otros contextos abordando las interseccionalidades de género.

\section{Conclusiones}

Este estudio analiza la victimización y perpetración de violencia en relaciones de pareja de las adolescentes de Bogotá-Colombia, así como otras variables sociodemográficas comparando al grupo que buscó apoyo social por esta violencia con el grupo que no buscó apoyo.

El estudio aporta información relevante para comprender cómo opera la violencia de género en relaciones de pareja adolescente y constituye el referente de partida al considerar por primera vez en Colombia al apoyo social como la variable dependiente. Según los hallazgos, la violencia responde a un continuum sustentado en la instauración de la dominación masculina a través del control, que se encuentra incorporada al imaginario social y legitima, desde sus inicios, lo permitido en la relación de pareja, invisibilizando o naturalizando muchas de sus manifestaciones, lo que disminuye la búsqueda de apoyo y la aísla de sus redes sociales, potenciales fuentes de apoyo. La victimización con mayor VGCP acumulada incrementó la búsqueda de AS, sin que fueran las mujeres de este grupo (Si-AS) quienes más informaran ejercer violencia sobre sus parejas agresoras (perpetración). Así, las adolescentes sufren mucha más violencia perpetrada por sus parejas de la que ejercen hacia 
sus parejas. El hecho de convivir con el agresor y en familias con cabeza de hogar masculina desestimuló la búsqueda de apoyo por la violencia.

La evidencia encontrada sugiere que el ejercicio de la violencia en las relaciones de adolescentes del contexto sociocultural colombiano difiere de las distinciones señaladas en la literatura internacional (especialmente de Norteamérica y Europa), y que en cambio coincide con estudios que identifican violencia de género en mujeres adultas. La evidencia sugiere que, debería incluirse a cualquier mujer indistintamente de la edad o de convivir o no en pareja en el estudio de la violencia de género en relaciones de pareja heterosexual en contextos socioculturales similares a Colombia

Tal recomendación podría atacar el subregistro del problema (al no bifurcar las prevalencias en dos estudios diferenciados), un cierto encubrimiento de sus dimensiones reales y las causas estructurales que lo sustentan. Finalmente, los hallazgos aquí presentados se constituyen como fundamentos para (re)pensar tanto el diseño de políticas y programas sociales específicos para abordar la violencia de género en relaciones de adolescentes, como de las prácticas profesionales en distintas áreas del Trabajo Social (juventudes, comunitario, educativo, etc.) para la prevención de la violencia y la promoción de relaciones amorosas saludables. Estas actuaciones profesionales deberían incluir a las redes sociales para concebir y poner en marcha estrategias de deconstrucción de las relaciones violentas (ahora denominadas por la juventud relaciones tóxicas), elemento clave para consolidar los futuros modos de relacionarse en pareja

Dado que algunos de los resultados de esta investigación parecieran divergentes con otros estudios, hace falta producir un mayor número de estudios nacionales con una perspectiva de género que den cuenta sobre las lógicas de victimización-perpetración.

\section{Referencias bibliográficas}

Acosta-Vélez, M. (2016). Comportamiento de la violencia de pareja. Colombia, 2015. Forensis 2015. Datos para la vida, 17(1), 291-352. Recuperado de https://www.medicinalegal.gov.co/documents/20143/49523/Violencia+de+pareja. pdf.

Albuquerque-Netto, L. de, Vasconcelos-Moura, M. A., Azevedo-Queiroz, A. B., Costa-Leite, F. M., \& Fernandes e Silva, G. (2017). Isolamento de mulheres em situação de violência pelo parceiro íntimo: Uma condição em redes sociais. Escola Anna Nery, 21(1), e20170007. doi: 10.5935/1414-8145.20170007.

Albuquerque-Netto, L. de, Vasconcelos-Moura, M. A., França-Araujo, C. L., NascimentoSouza, M. H. do, \& Fernandes e Silva, G. (2017). Social support networks for women in situations of violence by an intimate partner. Texto $\mathcal{E}$ Contexto - Enfermagem. 26(2). doi: 10.1590/0104-07072017007120015. 
Reina-Barreto

Ariza-Sosa, G. (2013). Contextos explicativos de la violencia en las relaciones de pareja en Medellín en el siglo XXI. En M. Barrios-Acosta (Ed.), Violencia en las relaciones de pareja (pp. 15-40). Bogotá: Universidad Nacional de Colombia.

Baragatti, D. Y., Carlos, D. M., Cruz-Leitão, M. N., Carvalho-Ferriani, M., \& Silva, E. M. (2018). Critical path of women in situations of intimate partner violence. Revista LatinoAmericana De Enfermagem, 26, e3025. doi: 10.1590/1518-8345.2414.3025.

Barrera, M. (1986). Distinctions between social support concepts, measures, and models. American journal of community psychology, 14(4), 413-445. doi: 10.1007/BF00922627.

Barrón-López, A. (1996). El apoyo social. Aspectos teóricos y aplicaciones. Madrid, España: Siglo XXI.

Bernal-Pinilla, D. Y., Hernández-Cuchumbe, B. P., y Akl-Moanack, P. M. (2016). Estudio y conceptos sobre la violencia durante el noviazgo. Misión Jurídica: Revista de derecho y ciencias sociales, 9(11), 273-287.

Bonilla-Algovia, E., y Rivas-Rivero, E. (2019a). Relación entre la exposición a la violencia de pareja y los malos tratos en el noviazgo. Psychologia, 13(1), 89-99. doi: $10.21500 / 19002386.3966$.

Bonilla-Algovia, E., y Rivas-Rivero, E. (2019b). Violencia en el noviazgo en estudiantes colombianos: relación con la violencia de género en el entorno. Interacciones, 5(3), e197. doi: 10.24016/2019.v5n3.197.

Bosch-Fiol, E. y Ferrer-Pérez, V. (2013). Nuevo modelo explicativo para la violencia contra las mujeres en la pareja: el modelo piramidal y el proceso de filtraje. Asparkía, (24), 5467.

Bourdieu, P. (2000). La dominación masculina. Barcelona: Editorial Anagrama.

Brock-Carneiro, J., Pereira-Gomes, N., Campos, L. M., Ferreira da Silva, A., Santos da Cunha, K., \& De Sousa-Conceição, D. M. (2019). Understanding marital violence: A study in grounded theory. Revista Latino-Americana de Enfermagem, 27, e3185. doi: 10.1590/1518-8345.3116.3185.

Camargo, E. (2019). Gender inequality and intimate partner violence in Bolivia. Revista Colombiana de Sociología, 42(2), 257-277. doi: 10.15446/rcs.v42n2.69629.

Capilla-Pérez, A., y Villadóniga-Gómez, J. (2004). Pioneros del Trabajo Social. Una apuesta por descubrirlos. Exposición bibliográfica. España: Universidad de Huelva.

Caplan, G. (1974). Support systems and community mental health: Lectures on concept development. New York: Behavioral publications.

Cardona-Cuervo, J., Carrillo-Cruz, Y. A., y Caycedo-Guió, R. M. (2019). La garantía de los derechos de la mujer en el ordenamiento jurídico colombiano. Hallazgos, 16(32), 83106. doi: 10.15332/2422409X.3265.

Castañeda-Salgado, M. P. (2008). Metodología de la investigación feminista. Guatemala: Centro de investigaciones interdisciplinarias en ciencias y humanidades CEIICHUniversidad Autónoma de México. 
Reina-Barreto

Cifuentes-Osorio, S. (2018). Comportamiento de la violencia de pareja. Colombia, 2017. Forensis 2017. Datos para la vida, 19(1), 256-299. Recuperado de https:/ / www.medicinalegal.gov.co/documents/20143/262076/Forensis+2017+Inte ractivo.pdf/0a09fedb-f5e8-11f8-71ed-2d3b475e9b82.

Cleto, M., Covolan, N., \& Signorelli, M. C. (2019). Mulheres-mães em situação de violência doméstica e familiar no contexto do acolhimento institucional de seus(as) filhos(as): $\mathrm{O}$ paradoxo da proteção integral. Saúde E Sociedade, 28(3), 157-170. doi: 10.1590/s010412902019170922.

Díaz, P. (1987). Evaluación del apoyo social. En: R. Fernández-Ballesteros (Coord.). El Ambiente: Análisis psicológico (pp. 125-149). Madrid: Ediciones Pirámide.

Espinoza-Moraga, S., Vivanco-Muñoz, R., Veliz-Burgos, A., y Vargas-Peña, A. (2019). Violencia en la familia y en la relación de pareja en universitarios de Osorno, Chile. Polis, 18(52), 122-139. doi: 10.32735/s0718-6568/2019-n52-1371.

Ferrer-Pérez, V., y Bosch-Fiol, E. (2019). El género en el análisis de la violencia contra las mujeres en la pareja: de la "ceguera" de género a la investigación específica del mismo. Anuario de Psicología Jurídica, 29(1), 69-76. doi: 10.5093/apj2019a3.

Fondo de Población de las Naciones Unidas-UNFPA. (2019a). Estrategia de igualdad de género del UNFPA 2018-2021. Recuperado de www.unfpa.org/sites/default/files/pubpdf/19-132_UNFPA_GenderStrategy-SP.pdf.

Fondo de Población de las Naciones Unidas-UNFPA. (2019b). Plan estratégico 2018-2021. Recuperado de unfpa.org/sites/default/files/pub-pdf/18-044_UNFPA-SP2018SP_2018-03-12-1244_1.pdf.

Garzón-González, R. (2018). Interseccionalidad y salud pública en el ámbito de las relaciones erótico-afectivas entre adolescentes en Colombia. Athenea Digital. Revista de pensamiento e investigación social, 18(3), e-2070. doi: 10.5565/rev/athenea.2070.

Gottlieb, B. (1985). Preventive interventions involving social networks and social support. In B. Gottlieb (Ed.), Social networks and social support (pp. 201-232). Beverly Hills, California: Sage Publications.

Gracia-Fuster, E., Herrero-Olaizola, J., y Musitu-Ochoa, G. (1995). El apoyo social. Barcelona: Promociones y Publicaciones Universitarias S.A.

Gracia-Leiva, M., Puente-Martínez, A., Ubillos-Landa, S., \& Páez-Rovira, D. (2019). Dating violence (DV): A systematic meta-analysis review. Anales De Psicología, 35(2), 300-313. doi: 10.6018/analesps.35.2.333101.

Guruge, S., Thomson, M. S., George, U., \& Chaze, F. (2015). Social support, social conflict, and immigrant women's mental health in a Canadian context: a scoping review. Journal of Psychiatric and Mental Health Nursing, 22(9), 655-667. doi: 10.1111/jpm.12216.

Heise, L. L. (1998). Violence Against Women: An Integrated, Ecological Framework. Violence Against Women, 4(3), 262-290. doi: 10.1177/1077801298004003002.

Hombrados-Mendieta, M. (2013). Apoyo Social. En M. Hombrados-Mendieta (Ed.), Manual de Psicología Comunitaria (pp. 121-143). Madrid: Síntesis.

House, J. (1981). Work stress and social support. Estados Unidos: Addison-Wesley. 
Reina-Barreto

Hoyos, C., y Benjumea, A. M. (2016). Medidas de protección a mujeres víctimas de violencia: análisis de la ley 1257 de 2008 y recomendaciones para su efectividad. Recuperado de https:/ / humanas.org.co/wpcontent/uploads/2020/10/2.Analisis_ley_1257_2008_recomendaciones2016.pdf.

Jaramillo-Jaramillo, C. A., Holguín-Osorio, H., y Ramírez-Ramírez, L. P. (2016). Representaciones sociales sobre la violencia recibida y ejercida en los noviazgos juveniles heterosexuales en el Valle de Aburrá-Colombia. Katharsis: Revista de Ciencias Sociales, (21), 156-191. doi: 10.25057/25005731.766.

Jennings, W. G., Okeem, C., Piquero, A. R., Sellers, C. S., Theobald, D., \& Farrington, D. P. (2017). Dating and intimate partner violence among young persons ages 15-30: Evidence from a systematic review. Aggression and violent behavior, 33, 107-125. doi: 10.1016/j.avb.2017.01.007.

Jiménez-Rodrigo, M. L., y Guzmán-Ordaz, R. (2015). El caleidoscopio de la violencia contra las mujeres en la pareja desde la desigualdad de género: una revisión de enfoques analíticos. Revista de Estudios Sociales, (54), 93-106. doi: 10.7440/res54.2015.07.

Lagarde, M. (2005). Para mis socias de la vida. Claves feministas para el poderío y la autonomía de las mujeres, los liderazgos entrañables y las negociaciones en el amor. Madrid: Horas y Horas.

Lagarde, M. (2006). Del femicidio al feminicidio. Desde el Jardín de Freud, (6), 216-225. Recuperado de revistas.unal.edu.co/index.php/jardin/article/view/8343/8987.

Lee, J., Pomeroy, E. C., \& Bohman, T. M. (2007). Intimate Partner Violence and Psychological Health in a Sample of Asian and Caucasian Women: The Roles of Social Support and Coping. Journal of Family Violence, 22(8), 709-720 doi: 10.1007/s10896-007-9119-6.

Lemos-Mena, E., y Echeverri-Calero, N. (2019). Comportamiento de la violencia de pareja en Colombia, año 2018. Forensis 2018. Datos para la vida, 20(1), 197-228. Recuperado de https:/ / www.medicinalegal.gov.co/documents/20143/386932/Forensis+2018.pdf/ be4816a4-3da3-1ff0-2779-e7b5e3962d60.

Lin, N. (1986). Conceptualizing social support. In N. Lin, A. Dean \& W. Ensel (Eds.), Social support, life events, and depression (pp. 17-30). Orlando, Estados Unidos: Academic Press.

López-Rodríguez, E. D. (2019). Políticas públicas de protección de la mujer y derechos humanos en Colombia. Zulia, Venezuela: Fondo editorial UNERMB; Universidad de Zulia. Recuperado de https://bonga.unisimon.edu.co/bitstream/handle/20.500.12442/4727/Politicas_Pu blicas_Protecci\%C3\%B3n_Mujer_PDF.pdf?sequence=1\&amp;isAllowed=y.

Lorente-Molina, B., y Luxardo, N. (2018). Hacia una ciencia del trabajo social. Epistemologías, subalternidad y feminización. Cinta moebio, (61), 95-109. doi: 10.4067/S0717-554X2018000100095.

Marthé-Manjarres, A. (2017). Comportamiento de la violencia de pareja en Colombia, año 2016. Forensis 2016. Datos para la vida, 18(1), 306-350. Recuperado de 
Reina-Barreto

https:/ / www.medicinalegal.gov.co/documents/20143/49526/Forensis+2016.+Dato s+para+la+vida.pdf.

McClennen, J. (2010). Social Work and family violence. Theories, assessment, and intervention. New York: Springer Publishing Company.

Medina-Núñez, I., y Medina-Villegas, A. (2019). Violencias contra las mujeres en las relaciones de pareja en México. Intersticios Sociales, (18), 269-302.

Montoya-Ruiz, A. M., Cruz-Torrado, B. K., y Leottau-Mercado, P. E. (2013). "Porque te quiero..." una mirada a la violencia basada en género en las relaciones de noviazgo en la ciudad de Cartagena de Indias. Ratio Juris, 8(16), 181-199. doi: 10.24142/raju.v8n16a7.

Mullender, A. (2000). La violencia doméstica. Una nueva visión de un viejo problema. Barcelona: Paidós.

Pachón, X. (2007). La familia en Colombia a lo largo del siglo XX. En Y. Puyana y M. H. Ramírez (Eds.), Familias, cambios y estrategias (pp. 145-159). Bogotá: Universidad Nacional de Colombia. Facultad de Ciencias Humanas; Alcaldía Mayor de Bogotá. Secretaría Distrital de Integración Social.

Pataquiva-Wilches, M. (2015). Violencia contra la mujer en el marco de las relaciones de pareja 2009-2014. En Instituto Nacional de Medicina Legal y Ciencias Forenses, Masatugó. Mujer que recibe lo malo para entregar lo bueno 2009-2014 (pp. 133-176). Bogotá: Diseñum Tremens.

Pereira-Gomes, N., Freire-Diniz, N. M., Araújo dos Reis, L., \& Lorenzini-Erdmann, A. (2015). The social network for confronting conjugal violence: Representations of women who experience this health issue. Texto $\mathcal{E}$ Contexto - Enfermagem, 24(2), 316-324. doi: 10.1590/0104-07072015002140012.

Queiroga-Souto, R., Guruge, S., Barbosa-Merighi, M. A., Pinto de Jesus, M. C., Egit, S., \& Knowles, L. (2016). Intimate partner violence among speaking immigrant adult Portuguese women in Canada. Revista Da Escola De Enfermagem Da USP, 50(6), 905912. doi: 10.1590/s0080-623420160000700005.

Ramírez-Rodríguez, M. H. (2000). Las mujeres y la sociedad colonial de Santa Fe de Bogotá. 17501810. Bogotá: Instituto Colombiano de Antropología e Historia-ICANH.

Redondo-Pacheco, J., Inglés-Saura, C., y García-Lizarazo, K. (2017). Papel que juega la edad en la violencia en el noviazgo de estudiantes de la Universidad Pontificia Bolivariana de Bucaramanga. Diversitas: perspectivas en Psicología, 13(1), 41-54. doi: 10.15332/s17949998.2017.0001.03.

Reina-Barreto, J. A. (2016). El Apoyo Social en las mujeres bogotanas víctimas de violencia doméstica. Implicaciones para el Trabajo Social. En D. Muñoz, E. Díez, N. Caparrós y C. Gimeno (Coords.), Respuestas transdisciplinares en una sociedad global: Aportaciones desde el Trabajo Social (pp. 1-27). La Rioja: Universidad de la Rioja. Recuperado de https:// publicaciones.unirioja.es/catalogo/online/CIFETS_2016/Monografia/pdf/ TC028.pdf.

Reina-Barreto, J. A. (2020). El Apoyo Social en la violencia de género en relaciones de pareja 
Reina-Barreto

heterosexual. Caso Bogotá-Colombia (Tesis doctoral). Universidad Complutense de Madrid, Facultad de Trabajo Social. Madrid, España. Recuperado de https:/ /eprints.ucm.es/id/eprint/64884/1/T42225.pdf.

Reina-Barreto, J. A., Criollo-Espín, C. A., y Fernández-D’Andrea, K. (2019). Apoyo social en la maternidad indeseada de estudiantes universitarias ecuatorianas: Análisis desde la perspectiva de género. PROSPECTIVA. Revista de Trabajo Social e Intervención Social, (27), 107-137. doi: 10.25100/prts.v0i27.6745.

Rey-Anacona, C. A. (2017). Diferencias por sexo y variables asociadas con las agresiones sexuales en el noviazgo en universitarios Colombianos. Psychologia, 11(1), 25-37. doi: 10.21500/19002386.3099.

Rey-Anacona, C. A., Martínez-Gómez, J. A., y Londoño-Arredondo, N. H. (2017). Diferencias entre adolescentes del área rural-urbana en malos tratos durante el noviazgo. Diversitas: Perspectivas en Psicología, 13(2), 159-168. doi: 10.15332/s17949998.2017.0002.02.

Richmond, M. (2008). Diagnóstico Social. (2 ed.). Madrid: Siglo XXI Editores de España.

Rodríguez-Fernández, R., y Ortiz-Aguilar, L. (2018). Violencia de pareja, apoyo social y conflicto en mujeres mexicanas. Trabajo Social Hoy, (83), 7-26. doi: 10.12960/TSH.2018.0001.

Sánchez-Lara, M. (2015). Violencias de género. En PROFAMILIA, Encuesta Nacional de Demografía y Salud. Tomo II. Componente de salud sexual y salud reproductiva (pp. 395419). Bogotá: Profamilia.

Segato, R. L. (2003). Las estructuras elementales de la violencia: contrato y status en la etiología de la violencia. En: R. L. Segato, Las estructuras elementales de la violencia. Ensayos sobre género entre la antropología, el psicoanálisis y los derechos humanos (131-148). Bernal, Argentina: Universidad Nacional de Quilmes; Prometeo.

Segato, R. L. (2016). La guerra contra las mujeres. Madrid, España: Traficantes de sueños.

Simón-Gil, M. (sep, 2019). El Trabajo Social Forense, la valoración de lesiones y secuelas sociales en víctimas de agresión sexual y violencia de género. En I Congreso Nacional de la Asociación Española de Trabajadores Sociales Forenses “La perspectiva social en la justicia del siglo XXI". Universidad Rey Juan Carlos. Madrid, España.

Straus, M., Hamby, S., McCoy, S., \& Sugarman, D. (1996). The revised conflict tactics Scales (CTS2). Development and preliminary psychometric data. Journal of family Issues, 17(3), 283-316. doi: 10.1177/019251396017003001.

Vázquez-García, V., y Castro, R. (2011). “¿Mi novio sería capaz de matarme?” Violencia en el noviazgo entre adolescentes de la Universidad Autónoma Chapingo, México. Revista Latinoamericana de Ciencias Sociales, Niñez y Juventud, 6(2), 709-738. Recuperado de http://revistaumanizales.cinde.org.co/rlcsnj/index.php/RevistaLatinoamericana/article/view/241/125.

Vivanco-Muñoz, R, Espinoza-Moraga, S., Romo-Tregear, C., Véliz-Burgos, A., y VargasPeña, A. (2015). Perpetración y victimización de la violencia en relaciones de parejas 
en jóvenes que cursan educación superior en la ciudad de Osorno, Chile. POLIS, Revista Latinoamericana, 14(40), 1-15. doi: 10.32735/S0718-6568/2015-N40-1118.

World Health Organization. (2013). Global and regional estimates of violence against women: prevalence and health effects of intimate partner violence and non-partner sexual violence. Génova: World Health Organization.

Zamanillo-Peral, M. T. (2018). Epistemología del Trabajo Social. De la evidencia empírica a la exigencia teórica. Madrid: Ediciones Complutense. 


\section{OTROS ARTÍCULOS DE PROSPECTIVA No. 32 DE 2021}

\section{EDITORIAL}

Compromisos, dilemas y desafíos del Trabajo Social con dimensión colectiva en tiempos de pandemia

Enrique Pastor-Seller

\section{ARTÍCULOS}

La narrativa biográfica como alternativa para la comprensión del Conflicto y la violencia en Colombia: una experiencia pedagógica universitaria

Sara Sofía Castaño-Barco y Claudia BermúdezPeña

Experiencias de transición a la vida adulta de jóvenes que migraron solos. Un estudio en Aragón (España) Daniel Jiménez-Franco; Raquel Berzosa-Callén y Chabier Gimeno-Monterde

Fundamentals of Education in Intervention with Young Offenders. A Critical Review of a 'Successful' Case in Andalucía, España

David Herrera-Pastor

Subjetividad juvenil: lecturas desde y para el Trabajo Social en Colombia

Daniela Joya-Valbuena

Participación politica de los jóvenes del Valle del Cauca, Colombia, en la toma de decisiones públicas

Luis Eduardo Gutiérrez-Rojas y Amanda Ramírez-Giraldo

Victimización y perpetración de violencia en pareja adolescente y redes de apoyo en Colombia. Análisis con perspectiva de género

Johanna Alexandra Reina-Barreto
Apoyo social percibido, autoestima y maternidad adolescente: entre el respeto y la intrusión. Estudio en Traiguén, Chile

Gloria Mora-Guerrero, Luisa Escárate-Colín, Carol Espinoza-Lerdón y Andrea Peña-Paredes

El desarrollo en el Trabajo Social de Colombia: un campo transversal, disperso y polivalente

Maira Judith Contreras-Santos

Análisis de la situación laboral de las alimentadoras en la caficultura de la zona central colombiana, a la luz de la teoría de Nancy Fraser

Pablo Andrés Arango-Giraldo

Prioridades de investigación en la Obra social de empleados públicos, Mendoza, Argentina. Un estudio Delphi sobre producción de conocimientos en la seguridad social

Cecilia Amalia Molina

Producción de conocimiento y toma de decisiones. Relaciones entre academia y política pública para las familias en Colombia

José Raúl-Ruíz y Sandro Leonardo MunévarVargas

El staff de prácticas profesionales en Trabajo Social: espacio de fomento del aprendizaje autodirigido. Un estudio de caso en la Universidad Católica Luis Amigó, Colombia

Cristian Eduardo Blanco-García

\section{ARTISTA INVITADO}

Margie Reinel-Aguilar Ao Cicadidae

Prospectiva

\section{PROSPECTIVA Revista de Trabajo Social e Intervención Social}

No. 32 • jul.-dic. 2021

e-ISSN: 2389-993X • Universidad del Valle 